\title{
A UNIFIED APPROACH TO MEASURABLE AND CONTINUOUS SELECTIONS
}

\author{
BY \\ G. MÄGERL ${ }^{1}$
}

\begin{abstract}
An abstract selection theorem is presented which contains as special cases-among others-the measurable selection theorem of Kuratowski and Ryll-Nardzewski, as well as the continuous selection theorem of Michael.
\end{abstract}

Introduction. Measurable and continuous selection theorems have found manifold applications in several fields of analysis and it therefore seems to be of some interest to have a unified approach to these two types of selections. The aim of this article is to suggest a method for such a common treatment, which yields a simultaneous proof of measurable selection theorems for measurable correspondences and continuous selection theorems for lower semicontinuous correspondences.

Since continuity of mappings and lower semicontinuity of correspondences may both be regarded as measurability with respect to certain systems of sets, which are here called pavings, the main result of this paper is stated as a theorem on measurable selections for measurable correspondences between a paved space and a metric space, the latter carrying some additional structure needed to formulate the appropriate conditions.

This abstract result contains as special cases Michael's continuous selection theorem, the measurable selection theorem of Kuratowski and Ryll-Nardzewski, a continuous selection theorem of Bauer and Bear and some further results, most of which are due to Michael.

ACKNOWLEDgment. The author wishes to thank Professor D. Kölzow for his constant encouragement and support during the elaboration of this work.

Notation and terminology. In this paper, all topological spaces are assumed to be Hausdorff and all vector spaces are assumed to be over the reals. The closure of a subset $A$ of a topological space is denoted by $\operatorname{cl} A$, the convex hull and the set of extreme points of a subset $B$ of a topological linear space

Received by the editors October 17, 1977.

AMS (MOS) subject classifications (1970). Primary 28A05, 28A20, 54C60, $54 \mathrm{C65}$.

Key words and phrases. Measurable selections, continuous selections.

'This paper contains a part of the author's doctoral thesis written under supervision of Professor D. Kölzow at the University of Erlangen-Nürnberg. Its final version was prepared while the author visited the University of Washington, Seattle, supported by a grant from the German Academic Exchange Service. 
are denoted by conv $B$ and ex $B$ respectively. By a zero-dimensional space we mean a topological space with covering dimension zero.

If $X$ and $Y$ are sets and $\Phi$ is a subset of their Cartesian product $X \times Y$, we write $\Phi(x):=\{y \in Y:(x, y) \in \Phi\}$ for $x \in X$. If $\Phi(x)$ is nonempty for all $x$, then $\Phi$ is called a correspondence between $X$ and $Y$. Since in this case $\Phi$ can be identified with a map from $X$ to the nonempty subsets of $Y$, correspondences are often called set-valued or multi-valued maps in the literature. By abuse of notation, we often write " $\Phi: X \rightarrow Y$ is a correspondence" instead of " $\Phi$ is a correspondence between $X$ and $Y$ ". Let $\Phi: X \rightarrow Y$ be a correspondence. For $A \subseteq Y$ we denote by $\Phi^{-1}(A):=\{x \in X: \Phi(x) \cap A \neq \varnothing\}$ the preimage of $A$ under $\Phi$. A map $f: X \rightarrow Y$ is called a selection for $\Phi$ iff $f(x) \in \Phi(x)$ holds for all $x \in X$. If $X$ is a set and $\mathbb{Q}$ a collection of subsets of $X$, then $\mathscr{Q}$ is called a paving and $(X, \mathcal{Q})$ a paved space, if $\mathscr{Q}$ is closed under finite intersections and countable unions and if $X$ and $\varnothing$ belong to $Q$. The pavings we deal with are topologies, $\sigma$-algebras and classes of the form $\mathscr{F}_{\sigma}$, where $\mathscr{F}$ is an algebra $\left(\mathscr{F}_{\sigma}\right.$ is defined to be the collection of all countable unions of members of $\mathscr{F}$ ).

If $(X, \mathcal{Q})$ is a paved space, $Y$ a topological space, $\Phi: X \rightarrow Y$ a correspondence and $f: X \rightarrow Y$ a map, then $\Phi$ (resp. $f$ ) is called $\mathbb{Q}$-measurable, iff $\Phi^{-1}(G) \in \mathbb{Q}$ (resp. $f^{-1}(G) \in \mathbb{Q}$ ) holds for all open $G \subseteq Y$. If $\mathbb{Q}$ is a topology, an $\mathscr{Q}$-measurable correspondence is called lower semicontinuous and the $Q$-measurable maps are the continuous ones.

A pair $(C, \delta)$ is called a (geometric) complex, if $C$ is a subset of a linear space and $\delta$ is a covering of $C$ by finite dimensional simplices contained in $C$, such that $S \in \mathcal{S}$ implies that all faces of $S$ belong to $\mathcal{S}$, and $S, T \in \mathcal{S}$ implies that $S \cap T$ is a face of both $S$ and $T$ or empty.

Let $(C, \delta)$ be a complex. We denote by $V(C, \delta)$ its set of vertices (i.e. the set of $x \in C$ such that $\{x\}$ belongs to $\S)$ and call $\operatorname{dim}(C, \delta):=\sup \{\operatorname{dim} S$ : $s \in \delta\}$ its dimension. For $y \in C$ let $S(y)$ be the simplex of smallest dimension in $\mathcal{S}$, that contains $y$, and for $x \in V(C, \delta)$ we call $\operatorname{St}(x):=$ $\cup\{y \in C: x \in S(y)\}$ the star of $x . C$ is always assumed to be topologized by the finest topology inducing the Euclidean topology on each $S \in \mathcal{S}$ (this topology is called the Whitehead topology).

If $\mathcal{U}$ is a covering of a set $M$ a complex $N(\mathcal{U})$, called its (geometric) nerve is assigned to $\mathcal{Q}$ in the following way: For $U \in \mathcal{Q}$ let $e_{U} \in \mathbf{R}^{\mathcal{U}}$ be defined by $e_{U}(V)=0$ for $V \neq U$ and $e_{U}(U)=1$. Let $\delta(U):=\left\{\operatorname{conv}\left\{e_{V}: V \in\right.\right.$ $\left.\mathscr{U}^{\prime}\right\}: \mathscr{U}^{\prime} \subseteq \mathscr{U}, \mathscr{U}^{\prime}$ finite, $\left.\cap \mathcal{U}^{\prime} \neq \varnothing\right\}, \quad C(\mathcal{U}):=\cup \delta(U)$ and set $N(\mho):=(C(\mho), \delta(\mho))$.

1. Definitions and examples. In this section, those terms are defined that are used to describe appropriate conditions on the correspondence under consideration, as well as on its domain and its range to get the desired selection theorem. Each definition is followed by examples which indicate 
how concrete selection theorems will be deduced from the abstract result. For the remainder of the paper, $k$ is a cardinal number and $\alpha$ a nonnegative integer or $\infty$.

The first definition will lead to a condition for the domain of the correspondence.

DEFINITION 1. A paved space $(X, \mathcal{Q})$ is called $(k, \alpha)$-paracompact, iff every covering $\mathscr{B} \subseteq \mathbb{Q}$ of $X$ with card $\mathscr{B}<k$ admits a refinement $\mathscr{B}^{*} \subseteq \mathbb{Q}$, such that

(i) $\operatorname{dim} N\left(\mathscr{B}^{*}\right) \leqslant \alpha$,

(ii) there exists an $\mathcal{Q}$-measurable map $\phi: X \rightarrow N\left(\mathscr{B}^{*}\right)$ with $\phi^{-1}\left(\operatorname{St}\left(e_{B}\right)\right) \subseteq B$ for all $B \in \mathscr{B}^{*}$.

EXAMPLes. (1) Let $(X, \mathcal{Q})$ be a paved set. If $\mathcal{Q}$ is a $\sigma$-algebra or more generally if $\mathscr{F}$ is an algebra and $\mathbb{Q}=\mathscr{F}_{\sigma}$, then $(X, \mathcal{Q})$ is $\left(\aleph_{1}, 0\right)$-paracompact.

For the following examples, let $X$ be a topological space and $\mathbb{Q}$ the topology of $X$.

(2) If $X$ is paracompact, $(X, \mathscr{Q})$ is $(k, \infty)$ paracompact for arbitrary $k$.

(3) If $X$ is normal and countably paracompact, $(X, \mathcal{Q})$ is $\left(\aleph_{1}, \infty\right)$-paracompact.

(4) If $X$ is normal, $(X, \mathscr{Q})$ is $\left(\aleph_{0}, \infty\right)$-paracompact.

(5) If $X$ is Lindelöf and has a basis of open-closed sets for its topology, $(X, \mathbb{Q})$ is $(k, 0)$-paracompact for arbitrary $k$.

(6) If $X$ is paracompact and zero-dimensional, $(X, \mathcal{Q})$ is $(k, 0)$-paracompact for arbitrary $k$.

(7) If $X$ is normal, countably paracompact and zero-dimensional, $(X, \mathbb{Q})$ is $\left(\aleph_{1}, 0\right)$-paracompact.

(8) If $X$ is an arbitrary zero-dimensional space, $(X, \mathbb{Q})$ is $\left(\aleph_{0}, 0\right)$-paracompact.

Proof. For the proof of (1) and (5)-(8) we show that each covering $\mathscr{B} \subseteq \mathcal{Q}$ with cardinality as required has a disjoint refinement $\mathscr{B}^{*} \subseteq \mathcal{Q}$. Then $\operatorname{dim} N\left(\mathscr{B}^{*}\right)=0$ and $\phi: X \rightarrow N\left(\mathscr{B}^{*}\right)$ defined by $\phi(x):=e_{B}$ for $x \in B \in \mathscr{B}^{*}$ has the desired properties. For (6) and (7) this follows from Proposition 2 in [8], for (8) from the very definitions.

(1) Let $\mathscr{F}$ be an algebra, $\mathbb{Q}=\mathscr{F}_{\sigma}$ and $\mathscr{B}=\left\{B_{n}: n \in \mathbf{N}\right\} \subseteq \mathbb{Q}$ be a countable cover of $X$. For each $n \in \mathbf{N}$ there exists a sequence $\left(F_{m}^{n}\right)_{m \in \mathbf{N}}$ in $\mathscr{F}$ such that $B_{n}=\cup_{m=1}^{\infty} F_{m}^{n}$. Hence $\left\{F_{m}^{n}: n, m \in \mathbf{N}\right\}$ is a cover of $X$ by members of $\mathscr{F}$. Rearrange this family into a sequence $\left(F_{n}\right)_{n \in N}$ and set $B_{1}^{*}:=F_{1}, B_{n}^{*}:=F_{n} \backslash \cup_{i=1}^{n-1} F_{i}$ for $n \geqslant 2$. Since $\mathscr{F}$ is an algebra each $B_{n}^{*}$ belongs to $\mathscr{F} \subseteq \mathcal{Q} . B^{*}:=\left\{B_{n}^{*}: n \in \mathbf{N}\right\}$ is obviously a disjoint refinement of B.

(5) Let $\mathscr{B}$ be an open cover of $X$. By the assumptions on $X$ there exists a refinement $\mathfrak{B}^{\prime}$ of $\mathscr{B}$ consisting of sets which are both open and closed. By the Lindelöf property, there exists a countable subcover $\left\{B_{n}: n \in \mathbf{N}\right\}$ of $\mathscr{B}^{\prime}$. 
Letting $B_{1}^{*}:=B_{1}$ and $B_{n}^{*}:=B_{n} \backslash \cup_{i=1}^{n=1} B_{i}$ we end up with a disjoint open refinement $\left\{B_{n}^{*}: n \in \mathbf{N}\right\}$ of $\mathscr{B}$.

To prove (2) and (3), we start with an open cover $\mathscr{B}$ which is at most countable in case (3). In both cases we get an open, locally finite refinement

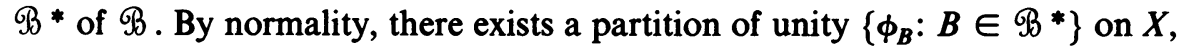
subordinated to $\mathscr{B}^{*}$. $\phi: X \rightarrow N\left(\mathscr{B}^{*}\right)$ defined by $\phi(x):=\Sigma_{B \in \mathscr{B}} \phi_{B}(x) \cdot e_{B}$ for $x \in X$ has the required properties (cf. [3, p. 172]). To prove (4) we simply observe that the geometric nerve of a finite cover has finite dimension and proceed as in (2) and (3).

RemarK 1. In fact, if $X$ is a Hausdorff space with topology $\mathbb{Q}$, then $(X, \mathbb{Q})$ is

zero-dimensional and normal, iff $(X, \mathcal{Q})$ is $\left(\aleph_{0}, 0\right)$-paracompact.

zero-dimensional, normal and countably paracompact, iff $(X, \mathcal{Q})$ is $\left(\aleph_{1}, 0\right)$ paracompact.

zero-dimensional and paracompact, iff $(X, \mathbb{Q})$ is $(k, 0)$-paracompact for all cardinals $k$. (Compare [10, pp. 109-110].)

The following definitions serve to formulate suitable conditions on the correspondence and its range.

Definition 2. (a) Let $Y$ be a set and $\mathscr{P}(Y)$ its power set. A map $H$ : $\mathscr{P}(Y) \rightarrow \mathscr{P}(Y)$ is called a hull-operator on $Y$ if $A \subseteq H(A)=H^{2}(A), H(A) \subseteq$ $H(B)$ for $A \subseteq B \subseteq Y$, and $H(\{y\})=\{y\}$ for $y \in Y$ holds.

(b) A hull-operator $H$ on a topological space $Y$ is called $\alpha$-convex, if the following is true:

For every complex $(C, \delta)$ with $\operatorname{dim}(C, \delta) \leqslant \alpha$ and every map $\rho: V(C, \delta) \rightarrow Y$ there exists a continuous map $\tau$ : $C \rightarrow Y$ such that $\tau(S) \subseteq H(\rho(\operatorname{ex} S))$ for all simplices $S \in$ א.

EXAMPLES. (9) The trivial hull-operator $A \mapsto A$ on a topological space is 0 -convex.

(10) The convex hull-operator $A \mapsto \operatorname{conv} A$ on a convex subset of a topological linear space is $\infty$-convex.

(11) Let $Y$ be a convex subset of a vector space and $d$ a convex metric in the sense of Bauer and Bear on $Y$ (i.e. the map $(\lambda, x, y) \mapsto \lambda x+(1-\lambda) y$ from $[0,1] \times Y^{2}$ onto $Y$ is continuous with respect to $d$, and for all convex $A \subseteq Y$ and $\varepsilon>0$ the set $\{y \in Y: d(y, A)<\varepsilon\}$ is convex). Then the convex hulloperator on $Y$ is $\infty$-convex.

Definition 3. (a) Let $Y$ be a set, $d$ a pseudometric on $Y$ and $H$ a hull-operator on $Y$.d is called $H$-convex if for all $A \subseteq Y$ with $A=H(A)$ and all $\varepsilon>0:\{y \in Y: d(y, A)<\varepsilon\}=H(\{y \in Y: d(y, A)<\varepsilon\})$.

(b) If $Y$ is a uniform space, a hull-operator $H$ on $Y$ is called compatible 
(with the uniform structure), if the uniformity of $Y$ is generated by a family of $H$-convex pseudometrics.

EXAMPLES. (12) Let $Y$ be a uniform space and $H$ the trivial hull-operator on $Y$. Then every pseudometric on $Y$ is $H$-convex and therefore $H$ is compatible.

(13) If $E$ is a vector space and $d$ a pseudometric on $E$ induced by a seminorm, then $d$ is $H$-convex, where $H$ is the convex hull-operator on $E$. Therefore, the convex hull-operator on a convex subset $Y$ of a locally convex space is compatible with the natural uniformity of $Y$.

(14) Let $Y$ and $d$ be as in example (11), then the convex hull-operator on $Y$ is compatible with the metric uniformity on $Y$.

REMARK 2. If $H$ is a compatible hull-operator on a metric space $(Y, d)$, the metric $d$ need not be $H$-convex (e.g. the convex hull-operator on a metrizable locally convex space $Y$ is compatible while even a translation-invariant metric on $Y$ is in general not $H$-convex for the convex hull-operator $H$ on $Y$ ).

Definition 4. A uniform space $Y$ is called $k$-bounded iff for any entourage $V$ of $Y$ there exists $Z \subseteq Y$, card $Z<k$, such that $Y=V(Z)$.

EXAMPLES. (15) A uniform space is totally bounded, iff it is $\aleph_{0}$-bounded.

(16) If a uniform space $Y$ contains a dense subset $Z$ with card $Z<k$, then it is $k$-bounded. In particular $Y$ is $k$-bounded for any $k>$ card $Y$.

2. An abstract selection theorem. In this section, we state and prove the abstract selection theorem. For the proof, we need two lemmas, the first showing how $(k, \alpha)$-paracompactness of paved spaces and $\alpha$-convexity of hull-operators are connected with selections.

LemMA 1. Let $(X, \mathcal{Q})$ be a $(k, \alpha)$-paracompact paved space and $H$ an $\alpha$-convex hull-operator on a topological space $Y$. Suppose $Z$ to be a subset of $Y$ with card $Z<k$ and $\{A(z): z \in Z\} \subseteq \mathbb{Q}$ to be a cover of $X$.

Then the correspondence $\Gamma: X \rightarrow Y$ defined by $\Gamma(x):=H(\{z \in Z: x \in$ $A(z)\})$ admits an $Q$-measurable selection.

Proof. Since $(X, \mathbb{Q})$ is $(k, \alpha)$-paracompact, there exist a refinement $\mathscr{B} \subseteq$ $\mathbb{Q}$ of $\{A(z): z \in Z\}$ with $\operatorname{dim} N(\mathscr{B}) \leqslant \alpha$ and an $\mathbb{Q}$-measurable map $\phi$ : $X \rightarrow N(\Re)$ such that $\phi^{-1}\left(\operatorname{St}\left(e_{B}\right)\right) \subseteq B$ holds for all $B \in \mathscr{B}$. Let $\nu: \Re \rightarrow Z$ be a map with $B \subseteq A(\nu(B))$ for $B \in \mathscr{B}$ and define $\rho: V(N(\mathscr{B})) \rightarrow Y$ by $\rho\left(e_{B}\right):=\nu(B)$. By the $\alpha$-convexity of $H$, we can extend $\rho$ to a continuous map $\tau: N(\Re) \rightarrow Y$ such that

$$
\tau(S) \subseteq H(\rho(\operatorname{ex} S))=H\left(\left\{\rho\left(e_{B}\right): e_{B} \in S\right\}\right)=H\left(\left\{\nu(B): e_{B} \in S\right\}\right)
$$

holds for each simplex $S$ of $N(\mathscr{B})$. Obviously $f:=\tau \circ \phi$ is an $\mathbb{Q}$-measurable map from $X$ into $Y$. To show that $f$ is a selection of $\Gamma$ let $x$ be an element of $X$ and $S:=S(\phi(x))$. Let $B \in \mathscr{B}$ be such that $e_{B} \in S$. Then $\phi(x) \in \operatorname{St}\left(e_{B}\right)$ and therefore $x \in \phi^{-1}\left(\operatorname{St}\left(e_{B}\right)\right) \subseteq B$. Hence we have: 


$$
\begin{aligned}
f(x) & =\tau(\phi(x)) \in \tau(S) \subseteq H\left(\left\{\nu(B): e_{B} \in S\right\}\right) \subseteq H(\{\nu(B): x \in B\}) \\
& \subseteq H(\{z \in Z: x \in A(z)\})=\Gamma(x) .
\end{aligned}
$$

The next lemma contains the essential idea to approximate the correspondence in an appropriate way by measurable mappings. It corresponds to a slightly extended version of Lemma (4.1) in [7] (or Lemma 1 in [8]) and to a part of the proof of the main theorem in [5].

Lemma 2. Let $(X, \mathfrak{Q})$ be a $(k, \alpha)$-paracompact paved space, $Y$ a $k$-bounded uniform space and $H$ an $\alpha$-convex hull-operator on $Y$. Let $d_{1}, d_{2}$ be continuous pseudometrics on $Y$ and $r, \varepsilon$ be positive real numbers. Assume that $\Phi: X \rightarrow Y$ is an $\mathcal{Q}$-measurable correspondence, and $g: X \rightarrow Y$ an $\mathbb{Q}$-measurable map, such that $d_{1}(g(x), \Phi(x))<r$ holds for all $x \in X$. Then there exists an $\mathbb{Q}$ measurable map $h: X \rightarrow Y$ such that for all $x \in X$ :

$$
h(x) \in H\left(\left\{y: d_{2}(y, \Phi(x))<\varepsilon\right\}\right) \cap H\left(\left\{y: d_{1}(y, g(x))<r+\varepsilon\right\}\right) .
$$

Proof. Let $d>\sup \left(d_{1}, d_{2}\right)$ be another continuous pseudometric on $Y$. Since $Y$ is $k$-bounded, there exists $Z \subseteq Y$, card $Z<k$, such that $d(y, Z)<$ $\varepsilon$ for all $y \in Y$. For $z \in Z$ let:

$$
\begin{aligned}
& C(z):=\Phi^{-1}\left(\left\{y: d_{2}(y, z)<\varepsilon\right\}\right)=\left\{x: d_{2}(\Phi(x), z)<\varepsilon\right\}, \\
& D(z):=g^{-1}\left(\left\{y: d_{1}(y, z)<r+\varepsilon\right\}\right)=\left\{x: d_{1}(g(x), z)<r+\varepsilon\right\},
\end{aligned}
$$

and $A(z):=C(z) \cap D(z)$. Since $\Phi$ and $g$ are $\mathbb{Q}$-measurable we have $A(z) \in$ $Q$ for all $z \in Z$ and we claim that $\{A(z): z \in Z\}$ is a covering of $X$.

Let $x$ be an element of $X$. By our assumptions on $\Phi$ and $g$ there exists $y \in \Phi(x)$ with $d_{1}(g(x), y)<r$. For $y$ choose $z \in Z$ such that $d(y, z)<\varepsilon$. Then we can conclude:

(a) $d_{1}(g(x), z) \leqslant d_{1}(g(x), y)+d_{1}(y, z)<r+d(y, z)<r+\varepsilon$,

(b) $d_{2}(\Phi(x), z) \leqslant d_{2}(y, z) \leqslant d(y, z)<\varepsilon$.

(a) implies $x \in D(z)$, (b) implies $x \in C(z)$, hence $x \in A(z)$.

Applying Lemma 1 , we get an $\mathbb{Q}$-measurable map $h: X \rightarrow Y$ such that $h(x) \in H(\{z \in Z: x \in A(z)\})$ for all $x \in X$. Hence we have for all $x \in X$ :

$$
\begin{aligned}
h(x) \in & H(\{z \in Z: x \in A(z)\}) \subseteq H(\{z \in Z: x \in C(z)\}) \\
& \cap H(\{z \in Z: x \in D(z)\})=H\left(\left\{z \in Z: d_{2}(\Phi(x), z)<\varepsilon\right\}\right) \\
& \cap H\left(\left\{z \in Z: d_{1}(g(x), z)<r+\varepsilon\right\}\right)
\end{aligned}
$$

and the proof of the lemma is complete.

With the aid of Lemma 2, we are able to prove the following abstract selection theorem:

THEOREM. Let $(X, \mathcal{Q})$ be a $(k, \alpha)$-paracompact paved space, $Y$ a $k$-bounded 
complete metric space and $H$ an $\alpha$-convex, compatible hull-operator on $Y$.

Then every $\mathbb{Q}$-measurable correspondence $\Phi$ between $X$ and $Y$ such that $\Phi(x)=\operatorname{cl} \Phi(x)=H(\Phi(x))$ admits an Q-measurable selection.

Proof. We may and do assume that the uniformity of $Y$ is generated by a sequence $\left(d_{n}\right)_{n}$ of $H$-convex pseudometrics, such that $d_{n+1} \geqslant 2 \cdot d_{n}$ holds for all $n$.

With the aid of Lemma 2, we will construct a sequence $\left(f_{n}\right)_{n}$ of $\mathcal{Q}$ measurable mappings from $X$ to $Y$ such that for all $n \geqslant 2$ and all $x \in X$ :

(i) $d_{n}\left(f_{n}(x), \Phi(x)\right)<1 / 2^{n}$,

(ii) $d_{n-1}\left(f_{n}(x), f_{n-1}(x)\right)<1 / 2^{n-2}$.

Assume for the moment that such a sequence is given. Then (ii) implies that this sequence is uniformly Cauchy and-since $Y$ is complete-converges to a map $f: X \rightarrow Y$. This map is $\mathbb{Q}$-measurable by uniform convergence of $\left(f_{n}\right)_{n}$, $\mathbb{Q}$-measurability of each $f_{n}$, metrizability of $Y$ and stability of $\mathbb{Q}$ under countable unions (cf. e.g. [4, Chapter 9, §43, No. XI, p. 267], or the lemma in [5]). From (i) follows that $f(x) \in \operatorname{cl} \Phi(x)=\Phi(x)$ holds for all $x$, i.e. $f$ is an $Q$-measurable selection of $\Phi$.

The sequence $\left(f_{n}\right)_{n}$ is constructed inductively as follows. Let without loss of generality the pseudometric $d_{1}$ be bounded by $\frac{1}{4}$. Let $f_{1}$ be a constant map from $X$ into $Y$. Since $X$ and $\varnothing$ belong to $\mathcal{Q}, f_{1}$ is $\mathcal{Q}$-measurable and obviously satisfies $d_{1}\left(f_{1}(x), \Phi(x)\right)<\frac{1}{2}$ for all $x \in X$. Applying Lemma 2, we get an $\mathcal{Q}$-measurable map $f_{2}: X \rightarrow Y$ such that

$$
f_{2}(x) \in H\left(\left\{y: d_{2}(y, \Phi(x))<\frac{1}{4}\right\}\right) \cap H\left(\left\{y: d_{1}\left(f_{1}(x), f_{2}(x)\right)<\frac{3}{4}\right\}\right)
$$

for all $x \in X$. Since $\Phi(x)=H(\Phi(x)),\{y\}=H(\{y\})$ for all $x \in X$ and $y \in Y$ and since $d_{1}, d_{2}$ are $H$-convex, we can omit the $H$ 's above and therefore (i) and (ii) hold for $n=2$.

Now let $n$ be at least 2 and $f_{1}, \ldots, f_{n-1}$ with the desired properties be given. Since $d_{n-1}\left(f_{n-1}(x), \Phi(x)\right)<1 / 2^{n-1}$ holds for all $x \in X$ we get-applying Lemma 2 once more- an $Q$-measurable $f_{n}: X \rightarrow Y$ such that

$$
\begin{aligned}
f_{n}(x) \in H\left(\left\{y: d_{n}(y, \Phi(x))<\frac{1}{2^{n}}\right\}\right) \\
\cap H\left(\left\{y: d_{n-1}\left(y, f_{n-1}(x)\right)<\frac{1}{2^{n-1}}+\frac{1}{2^{n}}\right\}\right) .
\end{aligned}
$$

As above, we may drop the $H$ 's and see that (i) and (ii) hold for $n$.

By induction, the proof of the theorem is complete.

3. Special cases of the abstract theorem. In this section, we show how several known selection theorems can be derived from our abstract theorem as special cases. 
(1) KuRATOWSKI AND RYLL-NARDZEWSKI [5]. Let $X$ be a set, $\mathscr{F}$ an algebra of subsets of $X$ and $\mathbb{Q}:=\mathscr{F}_{\sigma}$ the countably additive class generated by $\mathcal{F}_{\text {. Then }}$ every $\mathbb{Q}$-measurable correspondence between $X$ and a Polish space $Y$ such that $\Phi(x)$ is closed for all $x \in X$ admits an $\mathbb{Q}$-measurable selection.

In particular, every correspondence $\Psi: X \rightarrow Y$ such that $\Psi(x)$ is closed for all $x \in X$ and $\Psi^{-1}(F) \in \mathbb{Q}$ for all closed $F \subseteq Y$ has an $\mathbb{Q}$-measurable selection.

Proof. This follows from the abstract theorem by examples (1), (9), (12) and (16).

(2) Michael [7], [8]. Let $X$ be a paracompact topological space and $Y$ a convex subset of a locally convex linear space $E$. Let $\Phi$ be a lower semicontinuous correspondence between $X$ and $Y$ such that $\Phi(x)$ is closed and convex for all $x \in X$. Then each of the following conditions is sufficient for the existence of a continuous selection for $\Phi$.

(a) $E$ is a Fréchet space.

(b) The natural uniformity of $Y$ is complete and metrizable.

Proof. Examples (2), (10), (13) and (16) show that the assumptions of the theorem are satisfied in cases (a) and (b).

(3) BAUER AND BEAR [1]. Let $X$ be a paracompact topological space, $Y$ a convex subset of a linear space, completely metrized by a convex metric. Then every lower semicontinuous correspondence $\Phi: X \rightarrow Y$ such that $\Phi(x)$ is closed and convex for all $x \in X$ admits a continuous selection.

Proof. The assumptions of the abstract theorem are satisfied as examples (2), (11), (14) and (16) show.

(4) MichAel [7]. Let $X$ be a countably paracompact normal space, $Y$ be a convex subset of a locally convex space $E$ and $\Phi: X \rightarrow Y$ a lower semicontinuous correspondence such that $\Phi(x)$ is closed and convex for all $x \in X$. Then any of the following conditions implies that $\Phi$ has a continuous selection.

(a) $E$ is a separable Fréchet space.

(b) $Y$ is separable and its uniformity complete and metrizable.

Proof. Examples (3), (10), (13) and (16) show that the assumptions of the theorem are satisfied in cases (a) and (b).

(5) MichAEL [7], [9]. Let $X$ be a normal space and $Y$ a metrizable compact convex subset of a locally convex space. Then every lower semicontinuous correspondence $\Phi: X \rightarrow Y$ such that $\Phi(x)$ is closed and convex for all $x \in X$ admits a continuous selection.

Proof. The assumptions of the theorem are satisfied, by examples (4), (10), (13) and (15). 
(6) Let $X$ be a zero-dimensional topological space, $Y$ a complete metric space and $\Phi: X \rightarrow Y$ a lower semicontinuous correspondence, such that $\Phi(x)$ is closed for all $x \in X$. Then each of the following conditions implies that $\Phi$ has $a$ continuous selection.

(a) $Y$ is compact.

(b) MichaEL [7]. $X$ is paracompact.

(c) Michael [7]. $X$ is normal and countably paracompact and $Y$ is Polish.

Proof. The examples show that the assumptions of the abstract theorem are satisfied, namely: examples (8), (9), (12) and (15) in case (a), examples (6), (9), (12) and (16) in case (b), examples (7), (9), (12) and (16) in case (c).

(7) Let $X$ be a Lindelöf space with a basis of open-closed sets and $Y$ be a complete metric space. Then every lower semicontinuous correspondence $\Phi$ between $X$ and $Y$ such that $\Phi(x)$ is closed for all $x \in X$ admits a continuous selection.

Proof. Examples (5), (9), (12) and (16) show that the assumption of the abstract theorem are satisfied (or observe that (7) is a special case of (6)(b) as the proof following Example (5) shows).

RemarKs. (3) (2)(a) is not an immediate consequence of (3) by Remark 1.

(4) Obviously (3) remains true if it is only required that there exists a sequence of convex pseudometrics on $Y$, such that the uniformity they generate is separated and complete.

(5) Results analogous to (4) and (5) can be obtained in the context of (3) in an obvious manner.

(6) In (1), (2), (4), (6)(b) and (c) the completeness assumption on the whole space $Y$ can be omitted, if we require instead that each $\Phi(x)$ is complete. For replacing $Y$ by its completion, we are in the same situation as before.

(7) In (2), (4) and (5) the metrizability of $Y$ is essential as counterexamples by Corson and Lindenstrauss [2] and von Weizsäcker [11] show.

(8) (2) characterizes paracompactness in some sense. To be precise, the following theorem of Michael [7] holds: If $X$ is a Hausdorff topological space, $X$ is paracompact if the following is true:

For every Banach space $Y$ and every lower semicontinuous correspondence $\Phi: X \rightarrow Y$ such that $\Phi(x)$ is closed and convex for all $x$, there exists a continuous selection.

\section{REFERENCES}

1. H. Bauer and H. S. Bear, The part metric in convex sets, Pacific J. Math. 30 (1969), 15-33.

2. H. H. Corson and J. Lindenstrauss, Continuous selections with nonmetrizable range, Trans. Amer. Math. Soc. 121 (1966), 492-504.

3. J. Dugundji, Topology, 6th printing, Allyn and Bacon, Boston, Mass., 1970.

4. F. Hausdorff, Mengenlehre, de Gruyter, Berlin and Leipzig, 1927. 
5. K. Kuratowski and C. Ryll-Nardzewski, A general theorem on selectors, Bull. Acad. Polon. Sci. Ser. Sci. Astronom. Phys. 13 (1965), 397-403.

6. G. Mägerl, $Z u$ den Schnittsätzen von Michael und Kuratowski \& Ryll-Nardzewski, Dissertation, Erlangen, 1977.

7. E. Michael, Continuous selections. I, Ann. of Math. (2) 63 (1956), 361-382.

8. ___ Selected selection theorems, Amer. Math. Monthly 63 (1956), 233-238.

9.

10. A. R. Pears, Dimension theory of general spaces, Cambridge Univ. Press, Cambridge, 1975.

11. H. von Weizsäcker, Some negative results in the theory of lifting, in Measure Theory, Proc. Conf. Oberwolfach (A. Bellow, D. Kölzow, eds.), Lecture Notes in Math., vol. 541, SpringerVerlag, Berlin and New York, 1976, pp. 159-172.

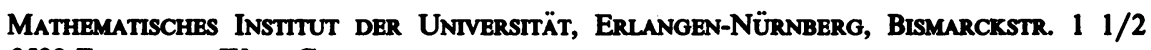
D-8520 Erlangen, West Germany

Department of Mathematics, University of Washington, Seattle, Washington 98195 